\title{
Short-course systemic corticosteroids in asthma: striking the balance between efficacy and safety
}

\author{
David Price (10) ${ }^{1,2}$, Mario Castro ${ }^{3}$, Arnaud Bourdin ${ }^{4}$, Sebastian Fucile ${ }^{5}$ and \\ Pablo Altman ${ }^{5}$
}

Affiliations: ${ }^{1}$ Observational and Pragmatic Research Institute, Singapore, Singapore. ${ }^{2}$ Centre of Academic Primary Care, Division of Applied Health Sciences, University of Aberdeen, Aberdeen, UK. ${ }^{3}$ Division of Pulmonary, Critical Care and Sleep Medicine, University of Kansas School of Medicine, Kansas City, MO, USA. ${ }^{4}$ Dept of Respiratory Diseases, Université de Montpellier, PhyMedExp, INSERM, CNRS, CHU de Montpellier, Montpellier, France. ${ }^{5}$ Novartis Pharmaceuticals Corporation, East Hanover, NJ, USA.

Correspondence: David Price, Observational and Pragmatic Research Institute (OPRI) Pte Ltd, 883 North Bridge Rd, \#02-05 Southbank, Singapore 198785. E-mail: dpricedopri.sg

@ERSpublications

Inappropriate use of systemic corticosteroids in asthma may add to disease burden. Even short-term, intermittent use is associated with health risks. Strategies to improve asthma control and reduce inappropriate use of systemic corticosteroids are needed. https://bit.ly/3bdieam

Cite this article as: Price D, Castro M, Bourdin A, et al. Short-course systemic corticosteroids in asthma: striking the balance between efficacy and safety. Eur Respir Rev 2020; 29: 190151 [https://doi.org/10.1183/ 16000617.0151-2019].

ABSTRACT Short courses of systemic corticosteroids (SCS), both oral and injectable, are very effective for the resolution of acute asthma symptoms, including exacerbations. However, the benefits of SCS, even short courses, must be balanced against the impact of their side-effects. While the adverse consequences of long-term use are widely recognised, there appears to be a perception in the medical community that short courses of SCS are safe. Limited but growing evidence in the literature suggests that even very brief dosing periods (3-7 days) of SCS are enough to cause significantly negative outcomes for patients. Short courses of SCS are associated with increased risk of adverse events including loss of bone density, hypertension and gastrointestinal ulcers/bleeds, in addition to serious impacts on mental health. Strategies to improve asthma control are recommended, including: 1) as-needed combination therapies in mild asthma; 2) risk factor reduction; 3) improving adherence/inhaler technique; 4) earlier initiation of add-on therapies; 5) use of biologics in appropriate patients; 6) development of new therapies to better control the disease; and 7) widespread education of the medical community. We propose that patients and primary care physicians should consider a cumulative SCS dose of $1 \mathrm{~g}$ per year as a highly relevant and easy-to-recall threshold.

\section{Introduction}

In 2015, asthma was listed as the most prevalent chronic respiratory disease in the world, affecting approximately 358 million people. It is likely that much of the burden associated with this disease is preventable or treatable with appropriate clinical and therapeutic interventions [1].

Since their efficacy in asthma was first described in the 1950s [2-4], use of systemic corticosteroids (SCS) in the management of this disease has become widespread. SCS are frequently prescribed in chronic airway diseases as both short- and long-term treatment options [5]. Short-course SCS are a very effective

Provenance: Publication of this peer-reviewed article was sponsored by Novartis, Switzerland (article sponsor, European Respiratory Review issue 155).

Received: 11 Nov 2019 | Accepted after revision: 20 March 2020

Copyright $\odot$ ERS 2020. This article is open access and distributed under the terms of the Creative Commons Attribution Non-Commercial Licence 4.0. 
and fast-acting option for the resolution of acute asthma symptoms including exacerbations [6]. Early administration of SCS for the treatment of severe asthma exacerbations is considered a standard of care and is recommended worldwide to be given to the patient within $1 \mathrm{~h}$ of presentation [7]. The Global Initiative for Asthma (GINA) recommends SCS for short-term (usually 5-7 days) treatment of severe acute exacerbations [8]. This represents an appropriate use of SCS.

Highly accessible and available over the counter in some countries, SCS are prescribed as rescue medication and are largely self-administered at home. It is noteworthy that SCS courses are used to treat not only severe, but also moderate and even mild exacerbations and symptoms [9].

While we recognise the obvious importance and effectiveness of SCS during asthma-related acuity, we also heed the importance of reducing the inappropriate use of SCS, as this can lead to potential deleterious effects. Thus, this article aims to raise awareness among the medical community of the potential side-effects associated with use of short courses of SCS in patients with asthma of any severity, as well as suggesting strategies to ensure their appropriate use.

\section{Use of SCS in asthma management}

SCS exert an anti-inflammatory effect in asthmatic airways by inhibiting the production of potent pro-inflammatory mediators and by reducing the chemotaxis of inflammatory cells to the lungs [10]. Possibly because of their efficacy, relative affordability or a perception that short courses are harmless, substantial over-prescribing of SCS has been reported in both adults and children with asthma $[9,11]$. Emergency SCS are prescribed as part of asthma self-management plans for patients at risk of exacerbations [12]; when not correctly implemented, this may lead to incorrect use of the medication, putting patients at risk of adverse events. Meanwhile, over-prescription of SCS may be an indicator of poor asthma control which can result from many factors, including poor adherence to inhaled medication (inhaled corticosteroids; ICS) and incorrect use of inhalers. In addition, patients with asthma may use SCS not only for asthma but also for frequently associated comorbidities that present as flaring-up diseases, for example rhinosinusitis with/without nasal polyps, atopic dermatitis, urticaria and conjunctivitis. SCS are widely prescribed for a variety of conditions, even where there is a lack of supporting evidence for their use [13]. An analysis of US national claims data found that more than one in five adults received at least one outpatient prescription for short-term ( $<30$ days) SCS over a 3-year period [14]. This suggests that SCS are seen by prescribers as an effective and cheap option, viewed as low-risk or "benign" medications. Patients with asthma often take SCS in addition to medium- or high-dose ICS, and sometimes also nasal corticosteroids, all of which are known to have systemic bioavailability. Cumulative side-effects of ICS use have been documented [15] and, therefore, patients taking both ICS and SCS may be at extra risk of steroid-related side-effects.

A recently published systematic review and meta-analysis by MAIJERS et al. [16] found that the SCS-sparing effect of high-dose ICS is mostly due to systemic effects. Based on the dose equivalence for effects on adrenal function, the authors of the review suggest that $1000 \mu \mathrm{g}$ fluticasone propionate has similar systemic effects to $5 \mathrm{mg}$ prednisone [17], and that $2500 \mu \mathrm{g}$ budesonide has similar systemic effects to $5 \mathrm{mg}$ prednisone [18]. It has been suggested that high doses of ICS should potentially be considered as harmful as low doses of SCS [19] and that they are accumulative on top of SCS.

The many systemic effects associated with long-term SCS use are well studied and described. The most common serious SCS-associated comorbidities include osteoporosis and osteopenia, type II diabetes, obesity, cardiovascular disorders and adrenal suppression. In addition, use of SCS has been associated with psychiatric symptoms such as insomnia, mania, anxiety or aggressive behaviour, dyspeptic disorder, hypertension, dyslipidaemia, infections, muscle atrophy, cataracts, glaucoma, bruising, change in physical appearance, skin striae and change in appetite [20-22].

Although the harmful side-effects of long-term use of SCS are widely known, there seems to be a perception in the medical community that short courses of SCS are safe provided they are used intermittently, rather than continuously. A nationwide cohort study in France reported that 59\% of patients with severe asthma were treated with SCS in 2012, with an average frequency of 3.3 courses per patient in the year [23].

Respiratory diseases are the most frequent indication for short-, medium- and long-term use of SCS, with a considerably higher frequency than other inflammatory conditions [5] and account for approximately $40 \%$ of total SCS prescriptions [24, 25].

There are risks associated with short-term intermittent use of SCS, as well as with longer-term use [26]. A recent report estimated that $93 \%$ of patients with severe asthma had at least one condition related to SCS exposure [27]. This includes morbidity and, more importantly, mortality. Regular SCS use is associated 
with greater all-cause mortality compared with non-SCS use [28, 29]. A nationwide asthma cohort study in Sweden found that patients with asthma who had regular SCS use had a 1.34-fold greater risk of death than those who did not use SCS [28]. The improvements in asthma symptoms seen with SCS, even short courses, must be balanced against the impact of the side-effects of this therapy.

\section{Side-effects of short-course SCS}

In this section we review recent publications on the side-effects of short-term use of SCS in the literature. These side-effects include a range of conditions that match those seen in long-term use of SCS (table 1). There is variability in the definition of "short courses" of SCS in the literature, making it difficult to comprehensively compare the adverse events associated with short-course SCS between studies [30]. GINA recommends a short course of SCS as typically $40-50 \mathrm{mg} \cdot \mathrm{day}^{-1}$ for 5-7 days [8]; previously published systematic reviews have defined a short course as $<14$ days [26] and other studies have categorised SCS courses of up to 30 days as short courses. Here we have included studies reporting SCS courses of up to 30 days.

The studies reviewed used database analyses to determine short course usage of SCS. This includes pharmacy claims, days of supply and number of prescriptions.

In a 4-year longitudinal study by MАтsumoто et al. [31], patients with asthma receiving $>2.5$ short courses of SCS (3-14 days) per year suffered a significantly larger loss in bone mineral density and had a significantly reduced Z-score (percentage of normal bone density predicted from age and sex) compared with patients who received $\leqslant 2.5$ courses in the year, highlighting that more than two courses of SCS in 1 year may negatively impact bone density [31]. This is an important concern due to the known detrimental link between low bone mineral density and increased risk for developing osteoporotic fractures [32].

A recent matched cohort study by PRICE et al. [20] in 48234 patients who were naïve to SCS at study entry compared the impact of SCS exposure on adverse events over a minimum of 2 years, using longitudinal medical record data drawn from the Optimum Patient Care Research Database (https://opcrd.co.uk) and the Clinical Practice Research Datalink (www.cprd.com). Patients in the SCS arm of the study received at least one additional prescription for SCS in the 18 months since their first SCS prescription. Compared with the non-SCS arm, patients with prior exposure to SCS had a significantly higher risk of developing an adverse event, including pneumonia, osteoporosis and fracture, type II diabetes, heart failure, cardio-/ cerebrovascular disease, cataract, myocardial infarction, sleep apnoea, renal impairment, depression/anxiety and body mass index (BMI) increase (table 2). Of significant importance, cumulative doses of SCS were associated with a clear dose-dependent increase in the risk of developing an adverse event in this study, with the equivalent of just four short SCS courses over a lifetime associated with harmful effects [20]. The dose-response relationship for cumulative SCS exposure with most adverse outcomes began at cumulative exposures of $1 \mathrm{~g}$ to $<2.5 \mathrm{~g}$ and for some outcomes at cumulative exposures of only $0.5 \mathrm{~g}$ to $<1 \mathrm{~g}$, equivalent to four lifetime SCS courses. It should be noted that this is the only one of the studies reviewed here that included a defined baseline or control group.

A 2017 retrospective cohort study in the USA assessed the frequency of short-course SCS prescriptions and the adverse events related to their use in adults using a nationwide dataset of private insurance claims ( $n=1548945$; not specifically limited to asthma). A high prevalence of short-course SCS prescriptions was observed in this study, with $21.1 \%(\mathrm{n}=327452)$ of patients receiving an outpatient prescription during a 3 -year period, and with $8.8 \%$ prescribed three or more courses. In this study, a short course was defined by a treatment period of $<30$ days. The duration of corticosteroid use was based on the "days' supply" variable provided within the pharmacy claim. Only sepsis, venous thromboembolism and fractures were specifically recorded in this study; however the prevalence of all three adverse events was significantly increased in patients with respiratory conditions receiving short-course SCS compared with those who did not (table 2) [14].

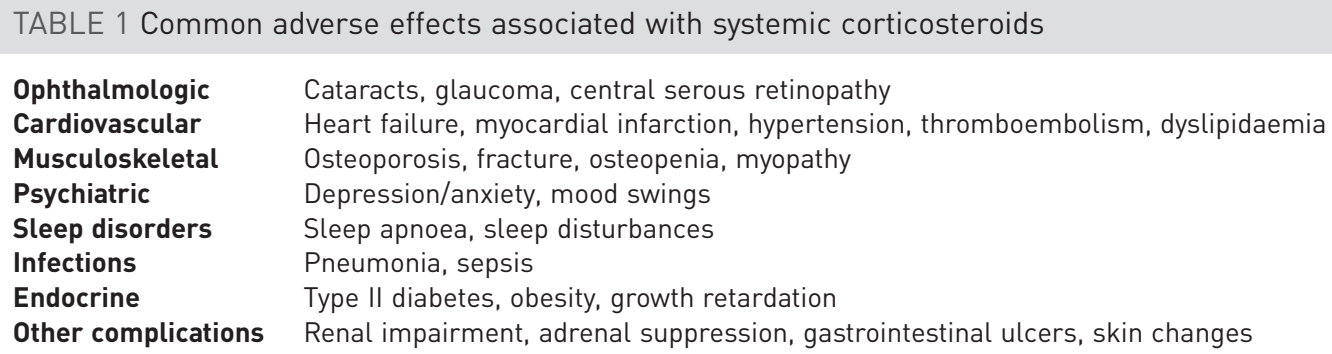

Ophthalmologic Cardiovascular Musculoskeletal Psychiatric Sleep disorders Infections

Endocrine

Other complications

Cataracts, glaucoma, central serous retinopathy

Heart failure, myocardial infarction, hypertension, thromboembolism, dyslipidaemia Osteoporosis, fracture, osteopenia, myopathy

Depression/anxiety, mood swings

Sleep apnoea, sleep disturbances

Pneumonia, sepsis

Type II diabetes, obesity, growth retardation

Renal impairment, adrenal suppression, gastrointestinal ulcers, skin changes

Data from [20-22]. 
TABLE 2 Reported risk of adverse outcomes associated with exposure to systemic corticosteroids (SCS)

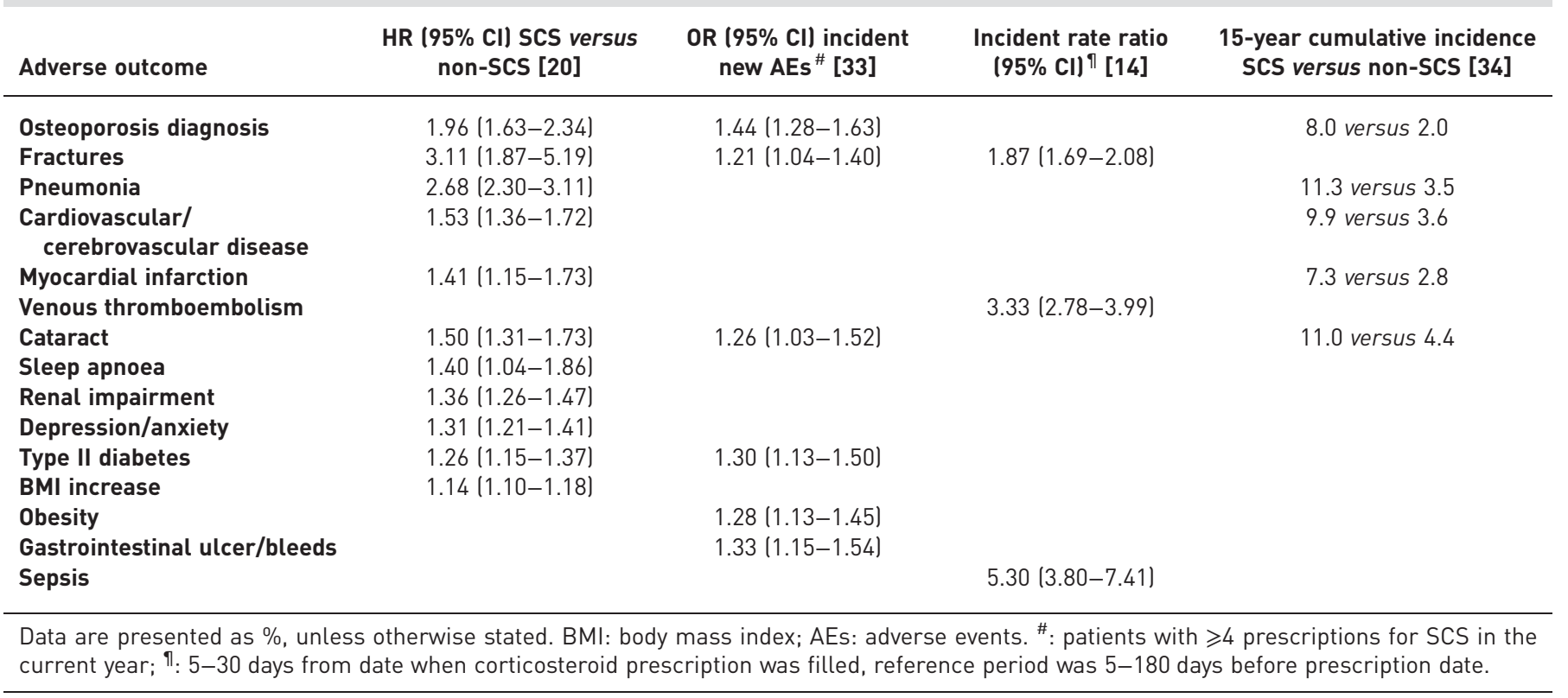

In a recent retrospective cohort study of the MarketScan insurance claims database by SulLIVAN et al. [33], patients receiving at least four intermittent SCS prescriptions in 1 year had a 1.29-times greater risk of experiencing an adverse event within the year compared with those in the non-SCS cohort. This study considered the number of prescriptions, regardless of dose or duration. Logistic regression analyses showed that even fewer SCS courses per year (one to three courses) remained significantly associated with the incidence of an adverse event [33]. There was greater likelihood of osteoporosis, fracture, hypertension, obesity, type II diabetes, gastrointestinal ulcers/bleeds and cataracts in patients receiving at least four SCS prescriptions in 1 year. The odds ratios for these adverse events was 1.21-1.44 (table 2). Indeed the risk of many of these adverse events remained statistically significant for one to three SCS courses in the year, also including fracture, hypertension and gastrointestinal ulcers/bleeds. This study also highlighted that the risk of experiencing an SCS-induced adverse event increased with each previous year of exposure to SCS. As well as demonstrating the adverse events associated with intermittent courses of SCS, these results show that the incidence of adverse events increased with each year of exposure, suggesting a cumulative toxic effect of short courses of SCS.

The onset of type II diabetes is one of the most common adverse outcomes related to SCS exposure [20] and there is evidence of a cumulative effect of increased exposure. Patients with asthma receiving an average daily SCS dose of $\geqslant 7.5 \mathrm{mg} \cdot$ day $^{-1}$ had a 15 -year cumulative incidence of type II diabetes (37.5\%) that was five times greater than those receiving $<0.5 \mathrm{mg} \cdot \mathrm{day}^{-1}$. Even three steroid bursts per year, over the long term, leads to increased risk of developing type II diabetes [34]. Weight gain, also a side-effect of SCS use, has been shown to worsen glycaemic control and increase the risk of diabetes progression. Obesity is a risk factor for many comorbidities of type II diabetes including cancer, gastrointestinal disease, osteoarthritis, liver and kidney diseases, sleep apnoea, respiratory disease and depression [35]. This highlights the potential negative consequences of short-term SCS and how their use for certain patients should be carefully considered.

Short-course SCS are widely used for the treatment of asthma in children; however, studies on the potential harm of these anti-inflammatory drugs are particularly limited for this patient cohort. A systematic literature review including 3200 patients aged $<18$ years of age (not specifically limited to treatment of asthma) revealed that vomiting, mood swings/behavioural issues and sleep disturbance were three common adverse events caused by short courses of systemic corticosteroids (defined by a treatment period of $\leqslant 14$ days) in this patient population [26]. Most patients across three randomised controlled trials experienced significant biochemical hypothalamic pituitary adrenal axis suppression linked to SCS. This is a particularly important observation because hypothalamic pituitary adrenal axis suppression can lead to stress-induced acute adrenal crisis or growth impairment [26].

A cohort follow-up substudy of the Childhood Asthma Management Program trial demonstrated a sex-specific, dose-dependent decrease in bone mineral accretion and associated increased risk for 
osteopenia in boys who received at least five intermittent short courses of SCS during the 7-year observation period [36]. Osteocalcin is a protein found in abundance in bone with a key role in bone growth, and is an early predictor for the development of osteoporosis [37]. A study examining the effects of short-course SCS on bone mineralisation in children with asthma reported a transient decrease in serum osteocalcin after 5 days of treatment with SCS. Osteocalcin levels returned to normal within 30 days, but indicate that the effects of SCS last much longer than their administration period [38].

Studies of the pharmacokinetics and pharmacodynamics of systemically administered corticosteroids have suggested that the detrimental mineralocorticoid effects of glucocorticoid treatment are pronounced when the capacity of the type 2 hydrogenase $11 \beta-\mathrm{HSD}_{2}$ is exceeded [39]. Higher steroid doses might have enhanced undesired mineralocorticoid effects when administered as multiple doses because of the prolonged time that mineralocorticoid receptors are occupied.

Impact of short-course SCS on mental health

Such is the frequency of mental health issues in patients receiving SCS, that a treatment algorithm has been developed to identify and manage corticosteroid-induced psychiatric syndromes [40].

A study in outpatients with asthma revealed a substantial effect of short-course SCS on mood change. After only 3-7 days of prednisone therapy, significantly increased symptoms of mania and mood changes were reported by both physicians and patients [41]. In this study, a significant correlation was also reported between worsening symptoms of mania and the number of prior courses of SCS, again suggesting a cumulative effect of multiple courses of intermittent SCS.

Similarly, 8 days of SCS therapy in patients with ophthalmic complaints caused an organic mood disorder in a significant proportion of patients; approximately $30 \%$ of patients experienced hypomania and $10-12 \%$ experienced a depressive syndrome [42].

Patient perception of short bursts of SCS should also be considered. A qualitative study in adults with severe asthma revealed significant patient concerns about current and future side-effects of SCS, including embarrassment about side-effects which altered their physical appearance, such as weight gain [43]. Patients with asthma and parents/guardians of children with asthma often have strong concerns about the safety of short courses of SCS, a factor which may prohibit full adherence [44, 45].

To mitigate some of these side-effects of SCS, it is suggested to monitor bone mineral density, blood pressure, blood lipids and glucose levels and assessment for adrenal insufficiency; use of bisphosphonates is recommended for patients at risk of osteoporosis [46].

\section{Economic impact of SCS-induced morbidity in asthma}

While the benefits of short courses of SCS outweigh the risks in patients with acute asthma, we believe these therapies are not warranted in all patients who currently receive them. As well as the health risks of short-course SCS, the economic impact of their use should be noted. Although SCS are inexpensive medications, SCS-related comorbidities/complications pose a high cost to the health system. The direct cost of steroid-induced morbidity in asthma is of major interest and concern [47].

A recent study in the UK showed a significant association between the extent of corticosteroid exposure and the number of comorbidities in patients with asthma. Furthermore, healthcare costs increased with corticosteroid exposure. In this study, data from the Optimum Patient Care Research Database were analysed for patients who had received at least four short courses of SCS in the previous 2 years versus patients with milder asthma or without asthma. Estimated annual cost of corticosteroid-induced morbidity in asthma was nearly six times higher for patients with high- versus low-SCS exposure [47].

In an observational cohort study in Sweden (PACEHR), similar results were observed. Age-adjusted total annual cost of comorbidities associated with SCS use was three times higher in the regular SCS group $(\geqslant 5 \mathrm{mg}$ per day) compared to the non-SCS users and more than twice as high as in the periodic SCS group ( $<5 \mathrm{mg}$ per day). In this study, pneumonia, diabetes, hypertensive disease, ischaemic heart disease, heart failure, cerebrovascular disease, depression and osteoporosis were significantly more frequent amongst patients who regularly received SCS compared with periodic users, and the non-SCS group [48]. However, it should be acknowledged that the association between healthcare costs and SCS use is also a reflection of disease severity.

\section{Strategies to reduce SCS use}

We suggest that awareness of the potentially harmful effects of SCS, regardless of the dose, duration or frequency of administration, needs to be raised further among healthcare professionals. We propose that patients and primary care physicians should heed the annual cumulative dose of SCS as a highly relevant and easy to access red alert. A cumulative dose of $1 \mathrm{~g}$ per year can be considered as an easy-to-recall 
threshold; this is equivalent to four short courses of SCS at the usual doses for treating an asthma exacerbation, and studies have suggested that the prevalence of many corticosteroid-induced comorbidities is increased at doses beyond this level $[20,27,33]$. Patients with asthma may be also be prescribed SCS to treat nasal polyps or rhinosinusitis, or other comorbidities, and so we recommend the $1 \mathrm{~g}$ per year threshold as a total dose which should include prescription of SCS for any indication. It is likely that significant savings could be made if corticosteroid-avoidance strategies could be considered in day-to-day clinical practice. We believe that several steps should be taken to reduce the use of SCS [7]. While guidelines are clear on the use of SCS for treatment for severe exacerbations it would be useful to consider further measures to enforce the appropriate use, i.e. consider stringent recommendations for both short-term and chronic SCS use. Implementing strategies to improve asthma control may also prove helpful, such as: 1) as-needed combination therapies in mild asthma; 2) risk factor reduction; 3) improving adherence/inhaler technique [49]; 4) increasing ICS dose during exacerbations [50]; 5) earlier initiation of add-on therapies; 6) use of biologics in appropriate patients; and 7) development of new therapies to better control the disease and avoid acute asthma symptoms/exacerbations that will require SCS. Above all, widespread education of the medical community is needed. Furthermore, education of our patients on the cumulative side-effects of SCS and high-dose ICS is critical to engage them in the care process. All these measures would help to limit the use of SCS to appropriate patients.

\section{Conclusions}

We acknowledge the crucial role of SCS in the treatment of acute asthma exacerbations and the effectiveness of these therapies in appropriate patients. However, we also believe that it is important to avoid the inappropriate use of these treatments, in order to prevent SCS-induced morbidity in asthma. Striking this balance between efficacy and safety is paramount; otherwise patients with asthma bear not only the burden of the disease, but also that of steroid-related morbidities.

The evidence in the literature on the association between short-course SCS and harmful adverse events is strengthening. It is clear that even very brief dosing periods (3-7 days) of SCS are enough to cause significantly negative outcomes for patients. The relatively high healthcare costs associated with SCS use combined with the side-effects of these drugs highlights the unmet need for steroid-sparing therapies for the treatment of patients with asthma.

Inappropriate short-term use of SCS for treatment of mild exacerbations or symptoms of asthma should be recognised as a significant healthcare problem which can be addressed through education of the medical community, by maximising the use of other effective treatments for asthma to reduce the need for SCS, and by limiting the annual cumulative dose to $1 \mathrm{~g}$.

Acknowledgements: The authors thank Gillian Lavelle and Claire Twomey (Novartis Product Lifecycle Services, Dublin, Ireland) for providing scientific writing support for this article, which was funded by Novartis Pharma AG (Basel, Switzerland) in accordance with Good Publication Practice (GPP3) guidelines (www.ismpp.org/gpp3).

Conflict of interest: D. Price reports personal fees from Amgen and Thermofisher; grants and personal fees from AstraZeneca, Boehringer Ingelheim, Chiesi, Circassia, Mylan, Mundipharma, Novartis, Pfizer, Regeneron Pharmaceuticals, Sanofi Genzyme, Teva and Theravance; personal fees from Cipla, GlaxoSmithKline and Kyorin; grants from Respiratory Effectiveness Group and UK National Health Service; non-financial support from Efficacy and Evaluation Mechanism Programme and Health Technology Assessment, outside the submitted work. He also has stock/ stock options from AKL Research and Development Ltd which produces phytopharmaceuticals and owns $74 \%$ of the social enterprise Optimum Patient Care Ltd (Australia and UK) and 74\% of Observational and Pragmatic Research Institute Pte Ltd (Singapore). M. Castro reports grants from NIH, ALA and PCORI; grants and personal fees from AstraZeneca, Novartis, GSK and Sanofi Aventis; personal fees from Teva, Genetech and Regeneron; and other fees from Elsevier, outside the submitted work. A. Bourdin reports personal fees, non-financial support and other fees from Actelion, AstraZeneca, Chiesi Pharmaceuticals and Novartis; grants, personal fees and non-financial support from Boehringer Ingelheim; grants, personal fees and other fees from GSK; personal fees and other fees from Regeneron; personal fees and non-financial support from Roche and Teva; other fees from Gilead, outside the submitted work. He is an advisory board member for Actelion, AstraZeneca, Boehringer Ingelheim, Chiesi Pharmaceuticals, Gilead, GSK, Novartis, Regeneron and Teva. S. Fucile is a full-time employee of Novartis Pharmaceuticals and holds shares in the company. P. Altman is a full-time employee of Novartis Pharmaceuticals and holds shares in the company.

\section{References}

1 GBD Chronic Respiratory Disease Collaborators. Global, regional, and national deaths, prevalence, disabilityadjusted life years, and years lived with disability for chronic obstructive pulmonary disease and asthma, 19902015: a systematic analysis for the Global Burden of Disease Study 2015. Lancet Respir Med 2017; 5: 691-706.

2 Brown HM. Treatment of chronic asthma with prednisolone; significance of eosinophils in the sputum. Lancet 1958; 2: 1245-1247.

3 Carryer HM, Koelsche GA, Prickman LE, et al. Effects of cortisone on bronchial asthma and hay fever occurring in subjects sensitive to ragweed pollen. Proc Staff Meet Mayo Clin 1950; 25: 482-486.

4 CONTROLLED trial of effects of cortisone acetate in status asthmaticus; report to the Medical Research Council by the subcommittee on clinical trials in asthma. Lancet 1956; 271: 803-806. 
5 Benard-Laribiere A, Pariente A, Pambrun E, et al. Prevalence and prescription patterns of oral glucocorticoids in adults: a retrospective cross-sectional and cohort analysis in France. BMJ Open 2017; 7: e015905.

6 Rowe $\mathrm{BH}$, Spooner $\mathrm{CH}$, Ducharme FM, et al. Corticosteroids for preventing relapse following acute exacerbations of asthma. Cochrane Database Syst Rev 2001; 1: CD000195.

7 Bourdin A, Bjermer L, Brightling C, et al. ERS/EAACI statement on severe exacerbations in asthma in adults: facts, priorities and key research questions. Eur Respir J 2019; 54: 1900900.

8 Global Initiative for Asthma. Global Strategy for Asthma Management and Prevention. Date last updated: April 2019. Date last accessed: September 2019. https://ginasthma.org/pocket-guide-for-asthma-management-andprevention

9 Farber HJ, Silveira EA, Vicere DR, et al. Oral corticosteroid prescribing for children with asthma in a Medicaid managed care program. Pediatrics 2017; 139: e20164146.

10 Barnes PJ, Adcock IM. How do corticosteroids work in asthma? Ann Intern Med 2003; 139: 359-370.

11 Arabkhazaeli A, Vijverberg SJ, van der Ent CK, et al. High incidence of oral corticosteroids prescriptions in children with asthma in early childhood. J Asthma 2016; 53: 1012-1017.

12 Pinnock H. Supported self-management for asthma. Breathe 2015; 11: 98-109.

13 Dvorin EL, Ebell MH. Short-term systemic corticosteroids: appropriate use in primary care. Am Fam Physician 2020; 101: 89-94.

14 Waljee AK, Rogers MA, Lin P, et al. Short term use of oral corticosteroids and related harms among adults in the United States: population based cohort study. BMJ 2017; 357: j1415.

15 Israel E, Banerjee TR, Fitzmaurice GM, et al. Effects of inhaled glucocorticoids on bone density in premenopausal women. N Engl J Med 2001; 345: 941-947.

16 Maijers I, Kearns N, Harper J, et al. Oral steroid-sparing effect of high-dose inhaled corticosteroids in asthma. Eur Respir J 2020; 55: 1901147.

17 Masoli M, Weatherall M, Holt S, et al. Inhaled fluticasone propionate and adrenal effects in adult asthma: systematic review and meta-analysis. Eur Respir J 2006; 28: 960-967.

18 Aaronson D, Kaiser H, Dockhorn R, et al. Effects of budesonide by means of the Turbuhaler on the hypothalmic-pituitary-adrenal axis in asthmatic subjects: a dose-response study. J Allergy Clin Immunol 1998; 101: 312-319.

19 Bourdin A, Suehs C, Charriot J. Integrating high dose inhaled corticosteroids into oral corticosteroids stewardship. Eur Respir J 2020; 55: 1902193.

20 Price DB, Trudo F, Voorham J, et al. Adverse outcomes from initiation of systemic corticosteroids for asthma: long-term observational study. J Asthma Allergy 2018; 11: 193-204.

21 Younes AK, Younes NK. Recovery of steroid induced adrenal insufficiency. Transl Pediatr 2017; 6: 269-273.

22 Poetker DM, Reh DD. A comprehensive review of the adverse effects of systemic corticosteroids. Otolaryngol Clin North Am 2010; 43: 753-768.

23 Bourdin A, Fabry-Vendrand C, Ostinelli J, et al. The burden of severe asthma in France: a case-control study using a medical claims database. J Allergy Clin Immunol Pract 2019; 7: 1477-1487.

24 Choo XN, Pavord ID. Morbidity associated with oral corticosteroids in patients with severe asthma. Thorax 2016 71: 302-304.

25 van Staa TP, Leufkens HG, Abenhaim L, et al. Use of oral corticosteroids in the United Kingdom. QJM 2000; 93: $105-111$.

26 Aljebab F, Choonara I, Conroy S. Systematic review of the toxicity of short-course oral corticosteroids in children. Arch Dis Child 2016; 101: 365-370.

27 Sweeney J, Patterson CC, Menzies-Gow A, et al. Comorbidity in severe asthma requiring systemic corticosteroid therapy: cross-sectional data from the Optimum Patient Care Research Database and the British Thoracic Difficult Asthma Registry. Thorax 2016; 71: 339-346.

28 Ekstrom M, Nwaru BI, Hasvold P, et al. Oral corticosteroid use, morbidity and mortality in asthma: a nationwide prospective cohort study in Sweden. Allergy 2019; 74: 2181.

29 Bourdin A, Molinari N, Vachier I, et al. Mortality: a neglected outcome in OCS-treated severe asthma. Eur Respir J 2017; 50: 1701486.

30 Normansell R, Kew KM, Mansour G. Different oral corticosteroid regimens for acute asthma. Cochrane Database Syst Rev 2016; 5: CD011801.

31 Matsumoto $\mathrm{H}$, Ishihara K, Hasegawa T, et al. Effects of inhaled corticosteroid and short courses of oral corticosteroids on bone mineral density in asthmatic patients: a 4-year longitudinal study. Chest 2001; 120: 1468-1473.

32 Qaseem A, Forciea MA, McLean RM, et al. Treatment of low bone density or osteoporosis to prevent fractures in men and women: a clinical practice guideline update from the American College of Physicians. Ann Intern Med 2017; 166: 818-839.

33 Sullivan PW, Ghushchyan VH, Globe G, et al. Oral corticosteroid exposure and adverse effects in asthmatic patients. J Allergy Clin Immunol 2018; 141: 110-116.

34 Voorham J, Xu X, Price DB, et al. Healthcare resource utilization and costs associated with incremental systemic corticosteroid exposure in asthma. Allergy 2019; 74: 273-283.

35 Ross SA, Dzida G, Vora J, et al. Impact of weight gain on outcomes in type 2 diabetes. Curr Med Res Opin 2011; 27: 1431-1438.

36 Kelly HW, Van Natta ML, Covar RA, et al. Effect of long-term corticosteroid use on bone mineral density in children: a prospective longitudinal assessment in the Childhood Asthma Management Program (CAMP) study. Pediatrics 2008; 122: e53-e61.

37 El-Dorry G, Ashry H, Ibrahim T, et al. Bone density, osteocalcin and deoxypyridinoline for early detection of osteoporosis in obese children. Open Access Maced J Med Sci 2015; 3: 413-419.

38 Ducharme FM, Chabot G, Polychronakos C, et al. Safety profile of frequent short courses of oral glucocorticoids in acute pediatric asthma: impact on bone metabolism, bone density, and adrenal function. Pediatrics 2003; 111: 376-383.

39 Czock D, Keller F, Rasche FM, et al. Pharmacokinetics and pharmacodynamics of systemically administered glucocorticoids. Clin Pharmacokinet 2005; 44: 61-98.

40 Warrington TP, Bostwick JM. Psychiatric adverse effects of corticosteroids. Mayo Clin Proc 2006; 81: 1361-1367. 
41 Brown ES, Suppes T, Khan DA, et al. Mood changes during prednisone bursts in outpatients with asthma. J Clin Psychopharmacol 2002; 22: 55-61.

42 Naber D, Sand P, Heigl B. Psychopathological and neuropsychological effects of 8-days' corticosteroid treatment. A prospective study. Psychoneuroendocrinology 1996; 21: 25-31.

43 Foster JM, McDonald VM, Guo M, et al. "I have lost in every facet of my life": the hidden burden of severe asthma. Eur Respir J 2017; 50: 1700765.

44 Cooper V, Metcalf L, Versnel J, et al. Patient-reported side effects, concerns and adherence to corticosteroid treatment for asthma, and comparison with physician estimates of side-effect prevalence: a UK-wide, cross-sectional study. NPJ Prim Care Respir Med 2015; 25: 15026.

45 Smith N, Smith A, Wang A, et al. Physician and parent barriers to the use of oral corticosteroids for the prevention of paediatric URTI-induced acute asthma exacerbations at home. Paediatr Child Health 2017; 22: 190-194.

46 Ramsahai JM, Wark PA. Appropriate use of oral corticosteroids for severe asthma. Med J Aust 2018; 209: S18-S21.

47 Barry LE, Sweeney J, O'Neill C, et al. The cost of systemic corticosteroid-induced morbidity in severe asthma: a health economic analysis. Respir Res 2017; 18: 129.

48 Janson C, Lisspers K, Stallberg B, et al. Health care resource utilization and cost for asthma patients regularly treated with oral corticosteroids - a Swedish observational cohort study (PACEHR). Respir Res 2018; 19: 168.

49 Price DB, Roman-Rodriguez M, McQueen RB, et al. Inhaler errors in the CRITIKAL study: type, frequency, and association with asthma outcomes. J Allergy Clin Immunol Pract 2017; 5: 1071-1081.

50 McKeever T, Mortimer K, Wilson A, et al. Quadrupling inhaled glucocorticoid dose to abort asthma exacerbations. N Engl J Med 2018; 378: 902-910. 